\title{
Frequency of helicobacter pylori infection in gross morphology of gastric carcinoma
}

\begin{abstract}
Objective: To determine the frequency of Helicobacter pylori infection in gastric carcinoma patients attending a tertiary care hospital in Karachi.

Study design: Descriptive, cross sectional study

Place \& duration of study: Department of Histopathology (DDRL) Dow Diagnostic \& research lab, Dow University of Health Sciences (DUHS) from Feb 2015 to Sep 2015.

Material \& methods: This study included 92 gastric tissues in which 79 were gastric carcinoma tissues and 13 were control samples taken from sleeve gastrectomy. Gastric Carcinoma tissues were divided into two subgroups dependent andnon-dependent helicobacter pylori. For gross morphology of gastric carcinoma, the gross types were divided into four types, Fungating, Ulcerative, infiltrating and polypoid. SPSS was used to analyse data.

Results: Mean age of the sleeve gastrectomy sample was 46.9 years, 5 male and 8 female. In gastric carcinoma sample, the mean age of non-dependent H. Pylori gastric carcinoma was 53.8 years, 7 male, 01 female, mean age of dependent $\mathrm{H}$. pylori gastric carcinoma was 53.1 years, 44 male, 28 female. For gross morphology of gastric carcinoma, the Fungating tumor was $25 \%$ in dependent $\mathrm{H}$. Pylori gastric carcinoma and $6 \%$ innon-dependent gastric carcinoma. Ulcerated tumor, $56 \%$ in dependent $\mathrm{H}$. Pylori gastric carcinoma and no cases in non-dependent H. Pylori gastric carcinoma. Invasive tumour, $13 \%$ in dependent $\mathrm{H}$. Pylori while no cases were seen in non-dependent $\mathrm{H}$. Pylori. No polypoid tumour was found in dependent as well as non-dependent $\mathrm{H}$. Pylori gastric carcinoma.
\end{abstract}

Conclusion: H. Pylori is significantly present in gastric carcinoma lying in the age of 50-70 years. However, the presence of H. Pylori dependent carcinoma is high among male population. The Ulcerated type of gastric carcinoma was more common in dependent H. Pylori gastric carcinoma.

Keywords: helicobacter pylori, gastric carcinoma, sleeve gastrectomy
Volume 4 Issue 6 - 2016

\author{
Nazia Qamar,' Saleh Memon,, Qadir Bux, ${ }^{3}$ \\ Muhammad Faisal Fahim ${ }^{4}$ \\ 'Assistant Professor, Pathology, Al-Tibri Medical College, Isra \\ University Karachi campus, Pakistan \\ ${ }^{2}$ Professor of Pathology,Al-Tibri Medical College, Isra University \\ Karachi campus, Pakistan \\ ${ }^{3}$ Assistant Professor,Anatomy, Al-Tibri Medical College, Isra \\ University Karachi campus, Pakistan \\ ${ }^{4}$ Statistician, Quality Enhancement Cell \& Research, Al-Tibri Medical \\ College, Isra University Karachi campus, Pakistan
}

Correspondence: Muhammad Faisal Fahim, Department of Quality Enhancement cell \& Research (QEC\&R),Al-Tibri Medical College, Isra University Karachi campus, Old thana Malir, Gaddap Town, Karachi-Pakistan, Tel +92346-3160827,

Email faisalfahim88@hotmail.com

\section{Introduction}

Usually, gastric cancer prevalence and transience have fallen extremely over the past 70 years ${ }^{1}$ but in spite of current decline gastric cancer are the $4^{\text {th }}$ most frequent cancer and second principal cause of cancer related death worldwide., ${ }^{2,3}$ According to annual cancer registry report of Shaukat khanum hospital, Lahore from Jan 2014 to Dec 2014, the data reported that Stomach Cancer is the $10^{\text {th }}$ leading cause of death amongst all age group and both Genders. In adults $>$ 18 years of age Gastric Cancer is the $8^{\text {th }}$ most chief cause of cancer in Pakistan. In adult males gastric cancer is the $6^{\text {th }}$ principal cause of cancer in Pakistan in spite of female adults it is not included in top 10 malignancies of Pakistan. ${ }^{4}$ The distinct geographic disparity, time trends and the migratory effect on the gastric cancer incidence recommend that environmental or lifestyle factors are major contributors to the aetiology of disease. ${ }^{5}$ The most important development in the field has been the identification of helicobacter Pylori as an important etiologic factor in gastric carcinoma because of its role in the development of chronic gastritis. ${ }^{6-9}$ Helicobacter pylori may generate an atmosphere which is highly positive to produce carcinogenesis and share with other life style and environmental exposures. There is evidence that consumption of salty foods and N-nitrosocompound and low intake of fresh fruits and vegetable augment the threat of gastric cancer. Helicobacter pylori gastritis support the growth of nitro sating bacteria which catalyze the production of carcinogenic $\mathrm{N}$ nitro so compound. ${ }^{10}$ There is a high frequency of helicobacter pylori in the developing world where people have low socioeconomic status and live in a germ-infested environment. ${ }^{11}$ Grossly, the carcinoma of stomach has wide distinction: 1) polypoid tumor (type I),2) Fungating tumor (Type II),3) Ulcerated tumor ( Type III), 4) Deeply invasive tumor (Type IV) [2]. Fungating tumor grow mainly into lumen, plane, ulcerated and severely invasive tumor develop largely all the way through the wall of stomach. ${ }^{12,13}$ The present study was designed to find out the frequency of H. pylori infection in gross morphology of gastric carcinoma according to age and gender and among Karachi population. 


\section{Material \& methods}

This is a cross sectional study conducted in Dow Diagnostic and research Lab (DDRL) in collaboration with Al-Tibri medical college, Karachi from Feb 2015 to September 2015. Total of 92 gastric tissue samples were investigated along with Informed consent and Clinical bio data of patients. The study was approved by the Ethical committee of Dow University of health sciences (DUHS) and Isra University Hyderabad Sindh. Patients who had a history of gastrointestinal symptoms with clinical support of Gastric cancer were selected regardless of age and gender along with this for control samples, sleeve gastrectomy specimen were selected from the patient who had a history of obesity, regardless of age and gender. Biopsy samples were examined grossly and microscopically. Biopsy materials were fixed in 10\% neutral buffered formalin, embedded in paraffin and $5 \mu \mathrm{m}$ sections were set for biopsy materials and they were cut to perform subsequent staining. ${ }^{14} \mathrm{H} \& \mathrm{E}$, Giemsa Stain (for H. Pylori) were the staining techniques used for staining the slides for histopatholgical examinations. For Statistical analysis, Statistical package for social sciences (SPSS) version 20.0. All Categorical Variables were expressed in numbers and percentage while continuous variables were shown in Mean \pm SD.

\section{Results}

In order to investigate the frequency of helicobacter pylori infection in gastric carcinoma. We were selected 92gastric tissue samples, of these $79 \%$ samples were from gastric carcinoma while total $13 \%$ were control samples. Overall the samples were divided into three groups. First was Control group, second was Non-dependent H. Pylori gastric carcinoma while third was dependent H.Pylori gastric carcinoma. Controls as well as gastric cancer samples were investigated for gender and age (Graph 1). In order to find out the type of disease in relation with age we found that the mean value of normal tissue is $46.9 \pm 5.2$ and it is discover among the range of 35- 54 years. As we compare this relation with non-dependent H. Pylori gastric carcinoma tissue we find the mean value is $53.8 \pm 15.9$ among the range of $30-76$ years. Now we compare with another relationship that is dependent H. pylori gastric carcinoma tissue it revealed the mean value is $53.1 \pm 14.86$ and it is find among range of 28-97 years. Total of 5 samples were from males and 8 samples were from females. The mean value of the male patient was $(38 \%)$ and a mean value of the female patient was $(62 \%)$ (Table1).

In non dependent H. Pylori gastric carcinoma, only 8 out of 79 samples were non dependent H. Pylori gastric carcinoma. Total of 7 samples were males and 1 from female. The mean value of the male patient was $(88 \%)$ and the female was $(13 \%)$ (Table 1$) .71$ out of 79 patients were dependent H. Pylori gastric carcinoma. Total of 44 patients were male and the mean value was (62\%) and 28 patients were females and the mean value was $38 \%$ (Table 1). In the frequency of gross types of gastric carcinoma, we found only 16 large biopsy samples out of 79 samples, the rest of 63 samples were small biopsy samples. Therefore, gross morphological classification was possible only on these 16 samples. Bormann classification, is the most global classification used for categorize macroscopic appearance of gastric cancer the samples were divided into two groups, Groups B and Groups C. The group B was not dependent H. Pylori gastric Carcinoma and Group $\mathrm{C}$ was dependent Helicobacter pylori gastric carcinoma.
In these groups. Fungating tumor was $25 \%$ in dependent H. Pylori gastric carcinoma and $6 \%$ was not dependent gastric carcinoma. The Ulcerated tumor was 56\% in dependent H. Pylori gastric carcinoma and non significant in non dependent H. Pylori gastric carcinoma. The Invasive tumor was $13 \%$ in dependent H. Pylori gastric carcinoma while non significant in non dependent H. Pylori gastric carcinoma. No Polypoid tumor was found in dependent as well as non dependent H. Pylori gastric carcinoma (Table 2, Graph 1\&2, and Figure 1\&2).

Table I Relationship of gender and disease

\begin{tabular}{llll}
\hline \multirow{2}{*}{ Types of disease } & \multicolumn{2}{l}{ Gender } & Total \\
\cline { 2 - 3 } Normal & Male & Female & \\
\hline \multirow{2}{*}{ Non-dependent } & 5 & 8 & 13 \\
& $38.50 \%$ & $61.50 \%$ & $100.00 \%$ \\
Dependent & $87.50 \%$ & $12.50 \%$ & $100.00 \%$ \\
Total & 44 & 27 & 71 \\
& $62.00 \%$ & $38.00 \%$ & $100.00 \%$ \\
& 12 & 9 & 21 \\
\hline
\end{tabular}

Table2 Frequency of gross types of stomach carcinoma (\%age)

\begin{tabular}{lll}
\hline $\begin{array}{l}\text { Gross types } \\
\text { of stomach } \\
\text { carcinomas }\end{array}$ & $\begin{array}{l}\text { Group B non- } \\
\text { dependent H. } \\
\text { pylori gastric } \\
\text { adenocarcinoma (\%) }\end{array}$ & $\begin{array}{l}\text { Group C dependent } \\
\text { H.pylori gastric } \\
\text { adenocarcinoma (\%) }\end{array}$ \\
\hline Fungating & $6 \%$ & $25 \%$ \\
Ulcerative & $0 \%$ & $56 \%$ \\
Infiltrating & $0 \%$ & $13 \%$ \\
Polypoid & $0 \%$ & $0 \%$ \\
\hline
\end{tabular}

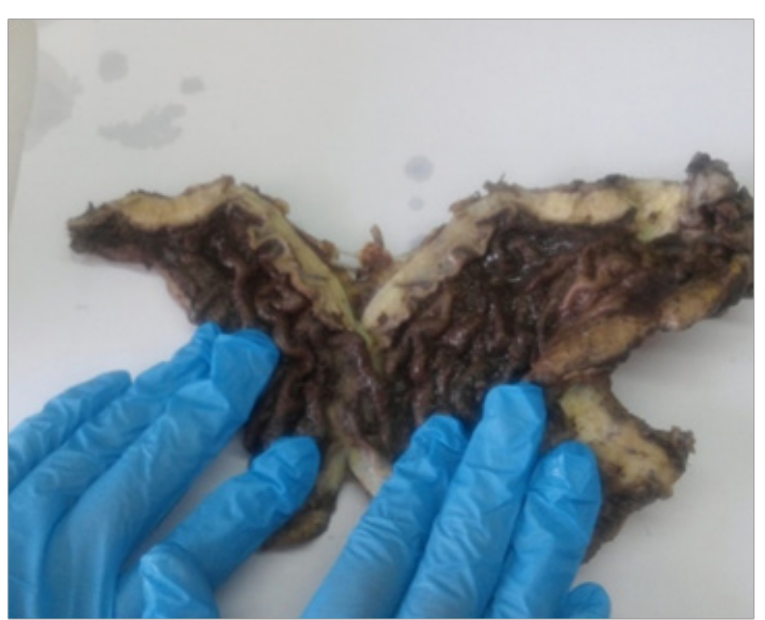

Figure I Ulcerated type of adenocarcinoma of stomach with heap up margins. 


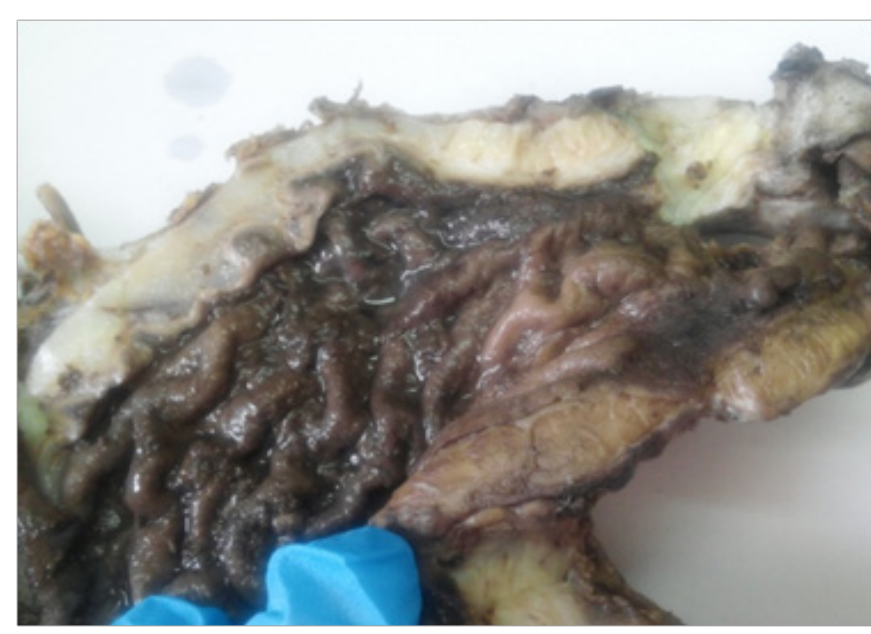

Figure 2 Infiltrative borders are present in the body of lesser curvature.

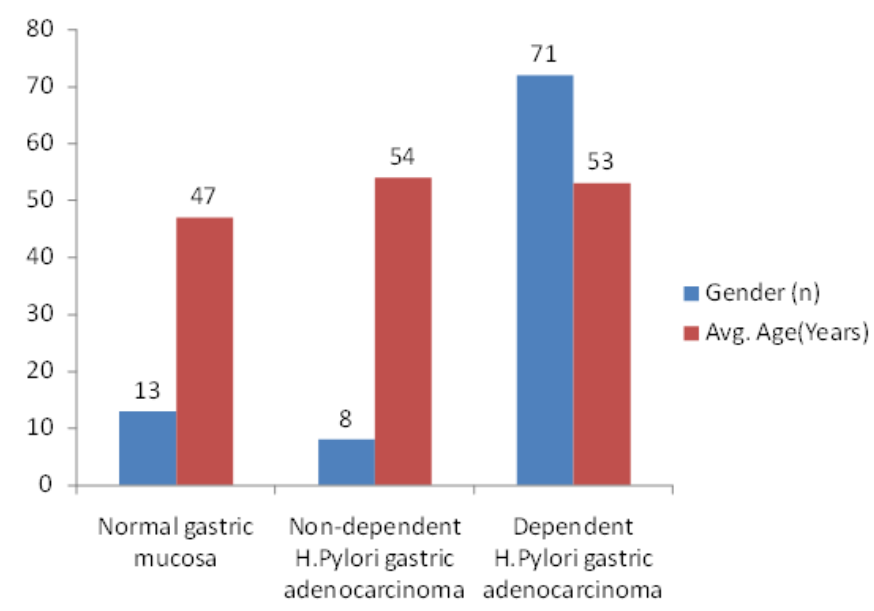

Graph I Bar chart showing overall age and gender distribution of normal gastric mucosa and gastric adenocarcinoma.

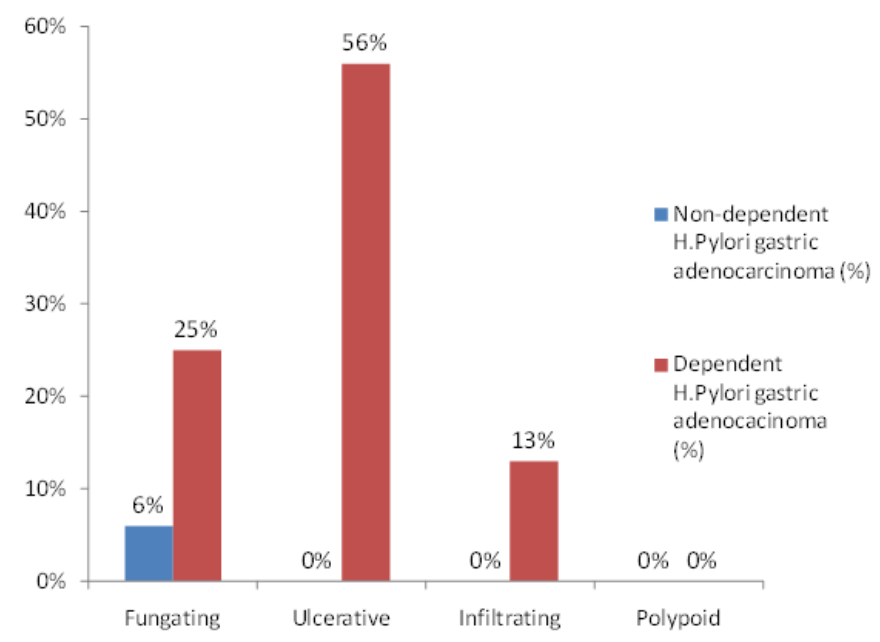

Graph 2 Bar Chart showing gross types of gastric adenocarcinoma.

\section{Discussion}

Gastric Cancer is the $4^{\text {th }}$ most frequently diagnosed cancer and it is the second most frequent cause of cancer related death worldwide. ${ }^{2,15}$ Gastric Cancer in Karachi fall into the standard of a low risk developing country pattern, but increasing incidence has been reported recently. ${ }^{16}$ Gastric carcinogenesis is a multifactorial process in which several factors are involved, some milieu lesions are also present, these lesions provide prospect to decrease the mortality rate of gastric carcinoma by earlier recognition of these lesions. ${ }^{17}$ Helicobacter pylori is a gram negative bacterium currently colonize the human stomach and also have been the centre of basic biochemical and clinical research since its innovation. ${ }^{18}$ This study addressed age and gender of patient as well as gross morphology of dependent and nondependent Helicobacter pylori in gastric carcinoma tissue as well as sleeve gastrectomy tissue, and according to our study, no significant difference noted between age of non dependent helicobacter pylori (54 years) and dependent helicobacter pylori (53 years) in gastric carcinoma. In other study conducted in Karachi, Pakistan, the mean age of dependent helicobacter pylori was found to be 49 years. ${ }^{19}$ In a study from Mexico, gastric cancer has been observed in persons aged between $50-70$ years. ${ }^{20}$ Cancer research statistics UK from 2011-2013 reported that highest number of new cases of gastric adenocarcinoma was present in between the age of 50-70 years. ${ }^{21}$ Our results coincide with other studies that reported the mean age of helicobacter pylori dependent gastric carcinoma was in between the range of (50-70 years). The reason for this non significant difference between the age of dependent and non dependent gastric carcinoma is there should be some other etiological factors in spite of $\mathrm{H}$. Pylori infection in the pathogenesis of gastric carcinoma in the range of this age groups in our society like tobacco use, sedentary life style etc. However, in our control group the age was one decade earlier than the gastric carcinoma groups because the control was taken from sleeve gastrectomy and the procedure is done in younger generation especially in female population experienced obesity. Most studies reported that gastric cancer occurs most frequently in male population. $^{22}$ In our study, male predominance was found in both dependent and nondependent H. Pylori gastric carcinoma that was concurring with other studies. Previous study in Pakistan revealed that gastric cancer occurs most frequently among males that was $60 \%$ in both type of cancer. ${ }^{19}$ In United States, an estimated 21, 320 cases of gastric cancer were reported and among these 13020 were males and 8300 were females and 10540 patient were die from this disease in 2012. ${ }^{23}$ Another study conducted in USA reported that men are affected twice as compared to women from gastric carcinoma. ${ }^{24}$ The reason for increase in frequency of gastric cancer among males in our society are possibly because of some etiological factors like life style factors like Pan tobacco use and smoking. Smoking is more prevalent in males. In this study we also found gross types of gastric carcinoma only in large biopsies which was less in number as compare to small biopsies. We found the prevalence rate of Ulcerative type was high that was $56 \%$ present in dependent H. Pylori gastric Carcinoma and Fungating type of tumor was $25 \%$ in dependent and $6 \%$ in non dependent gastric carcinoma, while invasive type was $13 \%$ in dependent H. Pylori gastric Carcinoma and Polypoid type was not found in our sample size. Our study regarding gross morphology of gastric carcinoma does not concur with other studies. In a previous study of USA report that the most common macroscopic type was a 
Fungating tumor which was found to be $36 \%$ and ulcerated was $25 \%$ and infiltrating is $26 \%$ and polypoid was $7 \% .^{25}$

\section{Conclusion}

Based on this study it is concluded that presence of Helicobacter pylori is significantly present in gastric carcinoma and it is considerably present between the ranges of 50-70 years while in sleeve gastrectomy the presence of helicobacter pylori is not very significant. However, the presence of $\mathrm{H}$. Pylori dependent carcinoma is high among male population. Grossly, the Ulcerated type of gastric carcinoma is high among dependent $\mathrm{H}$. Pylori gastric carcinoma.

\section{Acknowledgement}

None.

\section{Conflict of interest}

None.

\section{References}

1. Parkin DM, Pisani P, Ferlay J. Estimates of the worldwide incidence of eighteen major cancers in 1985. Int J Cancer. 1993;54(4): 594-606.

2. Parkin DM, Bray FI, Devesa SS. Cancer burden in the year 2000.The global picture. Eur J Cancer. 2001;37(Suppl 8):S4-S66.

3. Bing HU, Nassim El Hajj, Scott SIttler, et al. Gastric cancer: classification, histology and application of molecular pathology. $J$ Gastrointest Oncol. 2012;3(3):251-261.

4. Cancer registry and Clinical Data Management. Pakistan: SKMCH\&RC 2014.

5. Katherine D Crew, Alfred I Neugut. Epidemiology of Gastric cancer World J Gastroenterol. 2006;12(3):354-362.

6. Correa P. Helicobacter pylori and gastric carcinogenesis. Am J Surg Pathol. 1995; 19:S37-S43.

7. Koshida Y, Koizumi W, Sasabe M, et al. Association of Helicobacter pylori-dependent gastritis with gastric carcinomas in young Japanese patients: histopathological comparison of diffuse and intestinal type cancer cases. Histopathology. 2000;37(2):124-130.

8. Loffeld RJLF, Willems I, Flendrig JA, et al. Helicobacter pylori and gastric carcinoma. Histopathology. 1990;17:537-541.

9. Uemura N, Okamoto S, Yamamoto S, et al. Helicobacter pylori infection and the development of gastric cancer. $N$ Engl $J$ Med. 2001;345(11):784-789.
10. Sanduleanu S, Jonkers D, De Bruine A, et al. Non helicobacter pylori bacterial flora during acid suppressive therapy: differential findings in gastric juice and gastric mucosa. Aliment Pharmacol Ther. 2001;15(3):379-388.

11. Gill Hill, Majmudar P, Shankaran K et al. Age related prevalence of Helicobacter pylori antibodies in Indian subjects. Indian J Gastroenterol. 1994;13(3):92-94.

12. Correa P. Pathology of gastric cancer. Clin Oncol. 1984;3:251-257.

13. Stout AP. Tumors of the stomach. Section VI, fascicle 21 Atlas of tumor pathology, Washington DC. Armed Forces Institute of Pathology. 1953.

14. Qamar, Bukhari, Asrar, et al. Evaluation of Antral Gastric Biopsies A study of 50 patients at Mayo Hospital. Special Edition Annals. 2010;16(1):45-50.

15. Ferlay J, Shin HR, Bray F, et al. Estimates of worldwide burden of cancer in 2008. GLOBOCAN 2008. Int J Cancer. 2010;127(12): 2893-2917.

16. Taki K, Kuwabara N. Studies of histogenesis of the gastric carcinoma using minute cancers. Pathol Res Pract. 1981;172:176-190.

17. Haroon S, Faridi N, Lodhi Rashid Faisal. Et al. Frequency of precancerous lesions in endoscopic Gastritis Biopsies in Chronic Gastritis. Journal of college of physicians and surgeons of Pakistan. 2013;23(4):247-250.

18. Mohammad JS, Zaidi Faisal Syed, Toshiro Sugiyama. Epidemiological Ins and Outs of Helicobacter pylori: a review. J Pak med Assoc. 2012;62(9):955-959.

19. Muhammad A, Syed S. Association of helicobacter pylori with carcinoma of stomach. J Pak Med Assoc. 2007;57(7):337-341.

20. Horacio Noe Lopez Basev, Flavia Morales Vanques, Juan Manuel Ruiz Molina, et al. Gastric cancer in young people under 30years of age : worse prognosis or delay in diagnosis. Cancer Manag Res. 2013;5:31-36.

21. Cancer Research UK; 2008-2011.

22. Branum GD, Fink AS. Adenocarcinoma of stomach. In: DC Sabiston and Lyerly Hk (Ed). Textbook of Surgery WB Saunders, Philadelphia, Pennsylvania, USA. 1996;pp.893-907.

23. Siegal R, Naishadham, Jemal. Cancer statistics. CA Cancer J clin. 2012;62(1):10-29.

24. Dongyun Yang, Andrew Hendifar, Cosima Lenz, et al. Survival of metastatic gastric cancer:Significance of age, sex and race/ethinicity. $J$ Gastrointest Oncol. 2011;2(2):77-84.

25. Sun-Mi Lee, Kyung Mee kim, Jae YRo. Gastric Carcinoma: Morphologic Classifications and Molecular changes. In Tech. 2013;42:129-158. 